\title{
A quick method for evaluating the thresholds of workpiece surface damage in machining
}

\author{
Dongdong Xu a, d, Zhirong Liao a, Dragos Axinte (1) ${ }^{a,}$, , Mark Hardy b, Rachid M'Saoubi (1) c \\ ${ }^{a}$ Rolls-Royce UTC in Manufacturing and On-wing Technology, Faculty of Engineering, University of Nottingham, Nottingham,NG8 1BB, UK \\ ${ }^{b}$ Rolls-Royce plc, Derby, DE24 8BJ, UK \\ ${ }^{c}$ Seco Tools AB, R\&D Material and Technology Development, Fagersta, SE-73782, Sweden \\ ${ }^{d}$ Institute of Aerospace Technology, University of Nottingham, Nottingham, NG7 2TU, UK
}

This paper proposes a Pendulum-Based Cutting Test (PBCT) methodology which allows quick cutting tests for surface integrity evaluation along with providing cutting energies associated with particular level of workpiece surface damage; this is backed by an unified cutting energy model that links damage level of machined surface with energy partition in the cutting area. PBCT method could rapidly define the energy transferred to the workpiece that incurs particular magnitude of surface damage without using conventional machine tools and monitor the cutting process while only limited amount of materials is required. A demonstration of the proposed method is presented for Inconel718.

Surface integrity; Nickel alloy; Cutting energy

\section{Introduction}

The machinability of high strength aerospace materials (e.g. Nickel-based alloy) [1] decreases significantly with the increase of their strength at elevated temperatures; this becomes a challenge when ever-stronger new materials are developed. Surface integrity, as one of the critical aspects associated with machinability evaluation, is related to a few process parameters (e.g. tool geometry, cutting speed, depth of cut). With the varying combinations of cutting parameters, the machinability test becomes complicated due to the fact that multiple factors need to be considered in order to establish the threshold of cutting parameters that ensure the compliance with required workpiece surface integrity industrial standards. In particular, machinability vs. surface integrity is usually studied by conducting cutting tests on conventional machine tools by varying of cutting parameters followed by part sectioning to analyse superficial layer damage, which is time consuming and resourceful $[2,3]$ procedure. This is obviously more significant, especially when testing those materials on a laboratory scale when only a small batch of workpiece could be acquired.

One way to address the problem of performing extensive cutting tests is to define a particular "agent" which could map the surface integrity while reflecting the differences induced by various combinations of cutting parameters. Cutting energy, which is one of the features that reflect the phenomenon of machining process, has the potential to be employed as the "agent" for surface integrity. Although few research [4] have tried to stud the energy concept, most researches [5] focused on the study of consumed energy by the machine tools, which could, indirectly, reflect the cutting conditions but the information of specific damage on workpiece superficial layer has not been put in evidence.

To capture the consumed cutting energy in the cutting areas, a novel pendulum-based method is proposed in this paper in which the specific energies in different cutting zones could be evaluated thus, to enable the mapping of the workpiece surface integrity and establish the corresponding energy thresholds to ensure "damage-controlled" machined surfaces. In contrast, the pendulum grooving technique [6] and Split-Hopkinson Pressure Bar [7] tests that have been previously used only determining material properties (e.g. flow stress for modelling) or chip formation process rather than machinability evaluation and quick workpiece surface integrity evaluation as we propose here.

With the proposed pendulum-based cutting energy evaluation method, the machined surface integrity could be acquired and evaluated quickly by mapping the corresponding cutting energy against levels of workpiece damage rather than performing extensive tests with different cutting parameters on machine tools. The proposed PBCT method is quick and requires very limited amount of testing material while allowing the cutting process to be monitored in detail for deeper process understanding. To validate the proposed method, different cutting tool geometries were employed, while the consumed energy (shear, friction and ploughing energy) and corresponding surface integrity (by Scanning Electron Microscope-SEM, X-ray diffraction-XRD) were studied to allow the correlation between energy thresholds and levels of workpiece surface damages.

\section{Research concept and methodology}

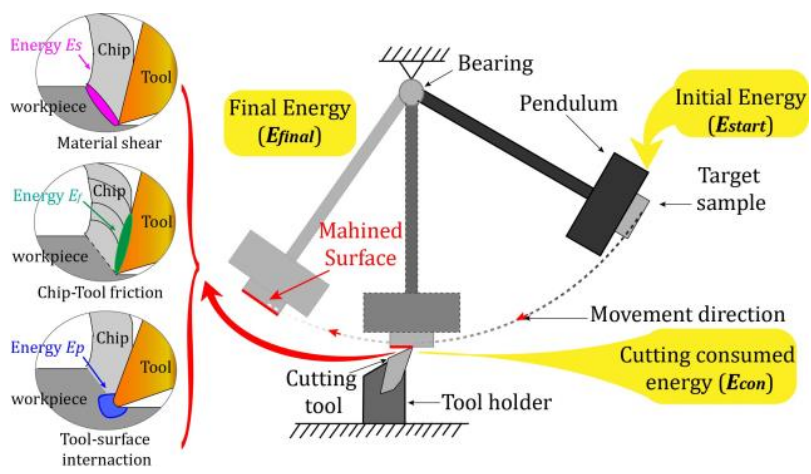

Fig. 1. Principle of cutting test in proposed method and energy footprint in cutting process

The proposed method for evaluating the thresholds of workpiece surface damage in machining was implemented on a 
Pendulum Based Cutting Test (PBCT) machine (Fig. 1), which consists of a variable-mass pendulum, to which the workpiece is attached, and a height-adjusted base on which the cutting tool is mounted. Varying the release angle of the pendulum allows the adjustment of both cutting speed $\left(v_{c}\right)$ as well as the start energy $\left(E_{\text {start }}\right)$ in the machining system.

Considering the PBCT setup as conservative system, an energybased surface integrity evaluation method was proposed that study the interaction of cutting tool, removed materials and generated machined surface, irrespective of different cutting parameters combination. That is, the consumed energy $\left(E_{c o n}\right)$ input to the cutting process (Fig. 1) is partitioned into areas: forming chips $\left(E_{s}\right)$, chip-tool interaction $\left(E_{f}\right)$ and tool-surface interaction $\left(E_{p}\right)$. Especially, as it will be proven later, the shear $\left(E_{s}\right)$ and ploughing $\left(E_{p}\right)$ energies could be directly associated with the level of superficial damage. Therefore, using our proposed approach, based on the relationship between surface integrity and consumed energy $\left(E_{s}, E_{f}, E_{p}\right)$ sets of cutting parameters that define thresholds of workpiece surface damage can be identified without the need of extensive cutting tests.

\subsection{Specialised setup for quick cutting tests}

The PBCT machine was designed and modified based on a standard Charpy machine where the swing hammer was replaced by a new designed variable-mass pendulum with a length of $350 \mathrm{~mm}$ and a starting mass of $5 \mathrm{~kg}$ to maximum weight of $10 \mathrm{~kg}$, as shown in Fig. 2. A thin Inconel 718 sample (e.g. $25 \times 20 \times 1 \mathrm{~mm}$ ) is fixed on the edge of the pendulum (Fig.2a). It is worth to note that a small chamfer was designed at the start of the sample to reduce the impact at the beginning of the cutting. A custom-made tool holder, mounted on a dynamometer fixed on the basement of the PBCT, was designed to accommodate various cutting edge geometries while allowing fine height adjustment (i.e. chip thickness). Different cutting speeds are obtained by adjusting the initial released angle of the pendulum (e.g. $\max v_{c}: 210 \mathrm{~m} / \mathrm{min}$ ).

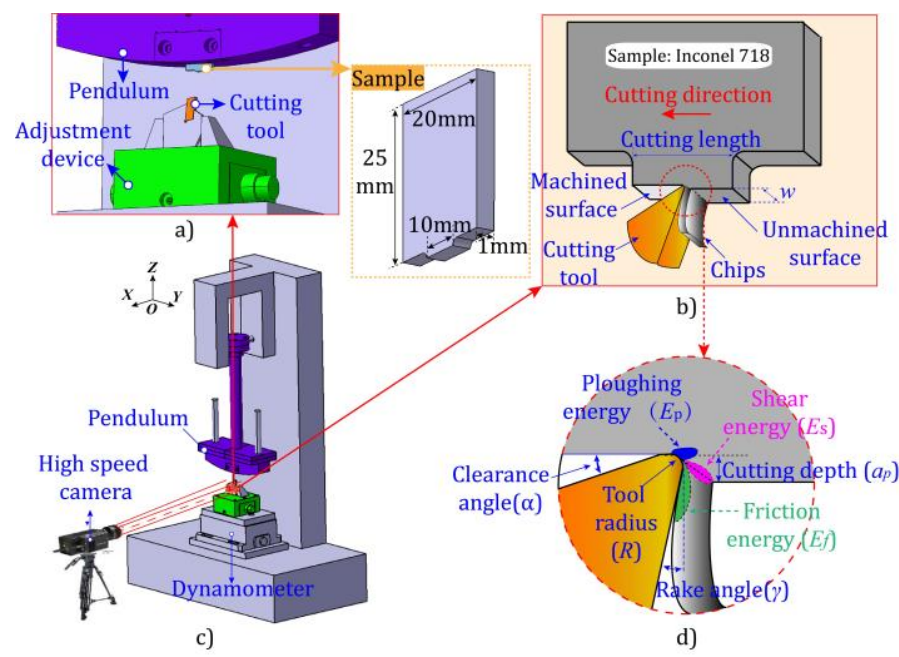

Fig. 2. Schematic of the designed PBCT machine

For this setup the cutting speed $\left(v_{c}\right)$ will continuously decrease from the start of cutting to its end and therefore, the consumed energies will also vary accordingly. Therefore, high speed camera is used to continuously monitor the cutting speed changing during the cutting process thus, allowing evaluation of cutting related energies. For those cutting conditions that require higher cutting energy (e.g. large cutting depth $\left(a_{p}\right)$ or width $(w)$, high strength materials), the input energy (from 0J to max. 60J) could also be adjusted by changing the mass weight of the pendulum.

\subsection{Governing energy models}

Specific cutting energy, which could be divided into shear energy $\left(E_{s}\right)$, friction energy $\left(E_{f}\right)$ and ploughing energy $\left(E_{p}\right)$, was analysed versus the machined surface formation process. The $E_{\text {con }}$ is the energy consumed in the cutting process after the impact. Therefore no impact energy is included and $E_{\text {con }}$ could be expressed as:

$$
E_{c o n}=E_{s}+E_{f}+E_{p}
$$

where $E_{s}$ is specific shear energy, $E_{f}$ is specific friction energy (between chips and tool rake face) and $E_{p}$ is specific ploughing energy (caused by tool edge radius). These energies could be calculated based on the formulas from the literature as shown in Table1, while the required data, (i.e. collected cutting force, cutting speed $\left(v_{c}\right)$, chip morphology and workpiece material property), could be acquired from the designed experiment setup. Although the $v_{c}$ varies in a single experiment, which contrasts with the conventional cutting tests, this does not affect the applicability of the proposed method since the corresponding cutting energies can be calculated in each measured point based on the tracking speed.

Table 1 Related formula for the calculation specific energies

\begin{tabular}{lcc}
\hline Energy type & Symbol & Corresponding literature \\
\hline Shear energy & $E_{s}$ & M.C. Shaw \&J.O. Cookson (2005)[8] \\
Friction energy & $E_{f}$ & M.C. Shaw \&J.O. Cookson (2005)[8] \\
Ploughing energy & $E_{p}$ & E. Budak et al. (2016)[9] \\
\hline
\end{tabular}

\section{Experimental setup}

As proposed in the present concept, the $E_{c o n}$ in the cutting process is aimed to be correlated with surface integrity and thus, to be an evaluation factor for subsurface damage level. To validate this, several cutting tests have been carried out on the proposed PBCT machine (Fig. 3). A Kistler 9257A dynamometer and NI 9223 data acquisition system were used to acquire the cutting force while high speed camera (IDT Y4) was employed to monitor (at $5000 \mathrm{~Hz}$ ) the cutting process and to capture the $v_{\mathrm{c}}$ in real-time. Chips were collected and measured for the calculation of $E_{c o n}$ while the machined surface integrity was studied using: SEM, to check workpiece subsurface damage (e.g. plastic deformation); and XRD to measure the residual stress profiles in the superficial layer. Tools with different edge radius $(R=$ sharp and rounded) and rake angles ( $\gamma=$ negative, neutral and positive) have been used (Table 2) with constant uncut chip thickness $\left(a_{\mathrm{p}}\right.$ $=0.1 \mathrm{~mm})$ and cutting width $(w=1 \mathrm{~mm})$, to achieve different levels of machined surface integrity as well as various specific cutting energies partition.

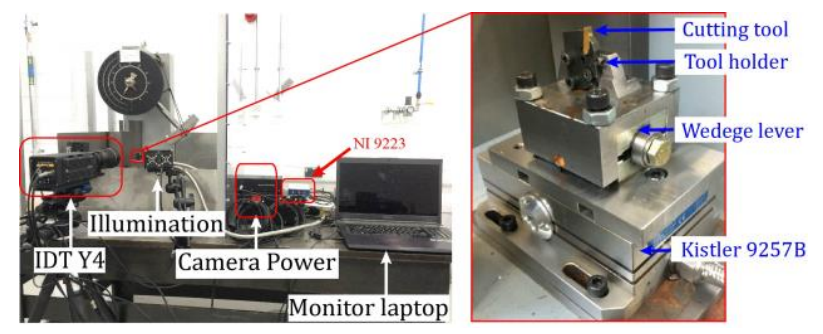

Fig. 3. Designed PBCT equipment and accessories

Table 2 Cutting conditions to study relationship between energy partition and surface integrity

\begin{tabular}{lcccccc}
\hline Test No. & No.1 & No.2 & No.3 & No.4 & No.5 & No.6 \\
\hline Edge type $(R)$ & & Rounded & & & Sharp & \\
Rake angle $(\gamma)$ & Neg & Neu & Pos & Neg & Neu & Pos \\
\hline
\end{tabular}

Note: Neg (Negative); Neu (Neutral); Pos (Positive) 


\section{Results and discussion}

\subsection{Experimental results}

By employing high speed camera the cutting process has been captured (Fig. 4) while the time decreasing cutting speeds determined with accuracy (see Fig. $5 \mathrm{a}$ and $5 \mathrm{~b}$ for all six tests). For instance, the $v_{\mathrm{c}}$ for the rounded tool with positive $\gamma$ in Fig. 5a varies from $70 \mathrm{~m} / \mathrm{min}$ and decreased to $39 \mathrm{~m} / \mathrm{min}$; similar tendency was observed for the other tests with higher speed deceleration when employing lower $\gamma$ (Neu and Neg) as they will consume more energy to remove same amount of material. The comparison of Fig.5a and Fig.5b, that higher end speed is found for sharp edge tools, shows that less energy was consumed when using smaller edge radius tools, which shows a good coherence with current finds [10].

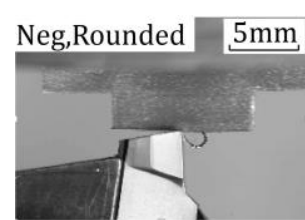

a)

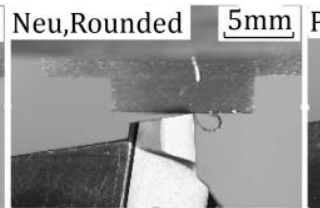

b)

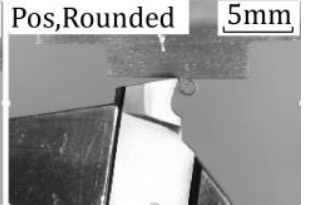

c)

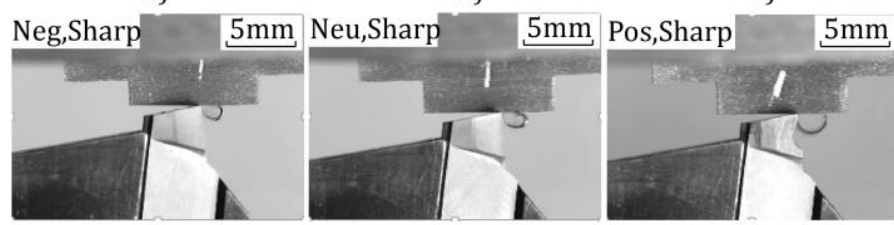

d) e) f)

Fig. 4. Selected frames for different cutting tools ( $\gamma$ and $R$ is varying)

Using these cutting speed diagrams a certain speed point (i.e. $v_{\mathrm{c}}=50 \mathrm{~m} / \mathrm{min}$ ) could be chosen for the comparison of the consumed energy between the different tests - see $t_{1}, t_{2}$ and $t_{3}$ in Fig. 5 a for test $1-3, t_{4}$, $t_{5}$ and $t_{6}$ in Fig. 5 b for test $4-6$. Then, the corresponding cutting forces at the chosen cutting speed (e.g. $v_{\mathrm{c}}=50 \mathrm{~m} / \mathrm{min}$ ) could be determined from the time dependent diagrams (Fig.5 c and Fig.5 d); for example for test 1-3 (Fig.5 a) where $v_{c}=50 \mathrm{~m} / \mathrm{min}$, the specific cutting forces (Fig. $5 \mathrm{c}$ ) are $3680 \mathrm{~N} / \mathrm{mm}^{2}$ (negative), $2790 \mathrm{~N} / \mathrm{mm}^{2}$ (neutral) and $2330 \mathrm{~N} / \mathrm{mm}^{2}$ (positive).

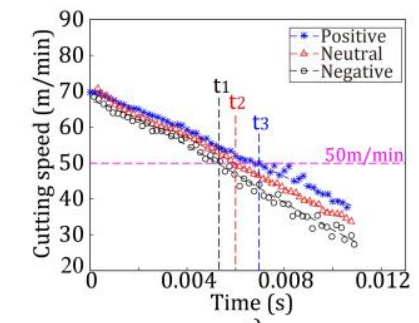

a)

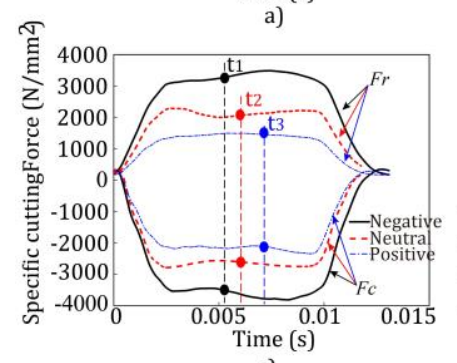

c)

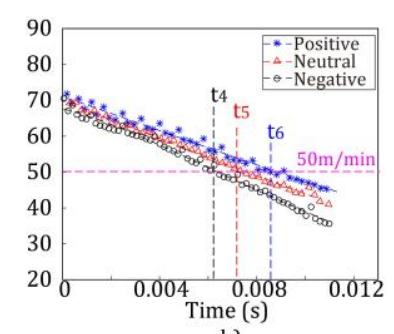

b)

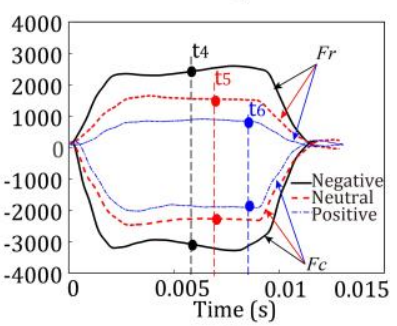

d)

Fig. 5. Cutting speeds and forces variation during cutting process: (a) and (c) test 1-3; (b) and (d) test 4-6

\subsection{Cutting energy consumption and partition}

Fig.6 reveals the main elements contributing to $E_{\text {con }}$ during the cutting process and their varying partition for tested cutting

conditions. For example, considering the test that uses a rounded tool with negative $\gamma, 55 \%$ of the $E_{\text {con }}$ was $E_{s}$ while $E_{f}$ was $39 \%$ and around $6 \%$ was $E_{p}$. With the increasing of $\gamma$ (from negative to positive), the proportion of $E_{s}$ starts to decrease while the $E_{f}$ has an increasing trend; at the same time the $E_{p}$ keeps almost constant during this process; and the same trends have been found for tools with sharp $R$. It is interesting to point out that the $E_{p}$ takes less than $1 \%$ and around $6 \%$ of $E_{c o n}$ for sharp and rounded tools, respectively.

Apart from that, this method is also able to capture the dependencies between consumed energy and cutting parameters. The energies (Fig.6b) (including $E_{\mathrm{con}}, E_{\mathrm{s}}, E_{\mathrm{f}}$ and $E_{\mathrm{p}}$ ) have similar decreasing tendency with $v_{c}$ that decreased with the increasing value of $\gamma$ (from negative to positive) for both sharp and rounded tools. This is because the shear strain is less and chips move away easily from the tool rake face when employing tools with bigger $\gamma$ (e.g. positive), therefore less force and energy will be needed for the cutting process. It could be summed up that the increasing of $\gamma$ has changed the energy partition (the percentage of shear increased significantly) in cutting areas and the changing of $R$ has a significant influence on the $E_{\mathrm{p}}$ variation (e.g. increased 10 times for rounded tools when compared with sharp edge).

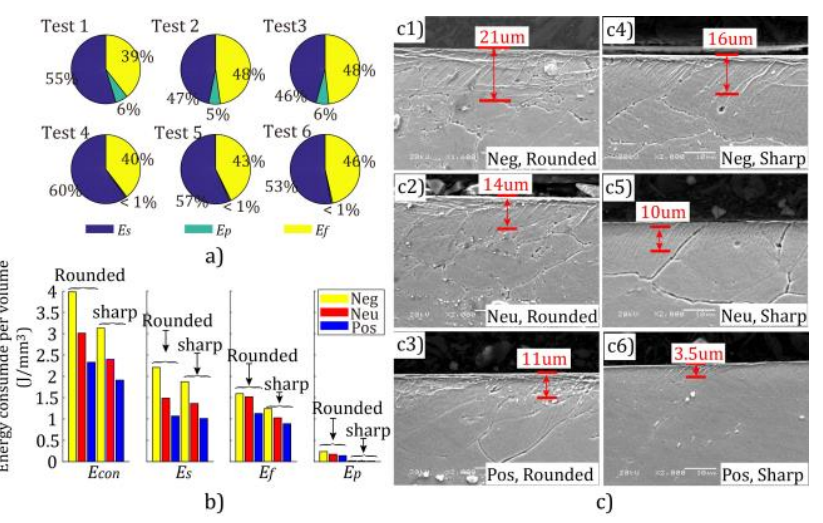

Fig. 6. Energy partition (a), and absolute energy partition (b) and surface integrity (c) during cutting process

\subsection{Relationship between consumed energy and surface integrity}

In order to correlate the consumed energies with the levels of surface damage, the workpiece surface integrity was studied with SEM, by evaluating the depth of swept grains (material drag) (Fig.6c). Clearly this method is able to capture the phenomenon that deformation depths for both sharp and rounded tools have similar decreasing trend with $E_{\text {con }}$ following the increasing of $\gamma$. For example, the deformation depth decreased from $21 \mu \mathrm{m}(\gamma$ =negative, Fig.6-c1) to $11 \mu \mathrm{m}$ ( $\gamma=$ positive, Fig.6-c3). Similar tendency was found from the residual stress profiles under the machined surfaces (Fig.7) which reveal that sharp tools result in shallower (percentage) stress affected layers.
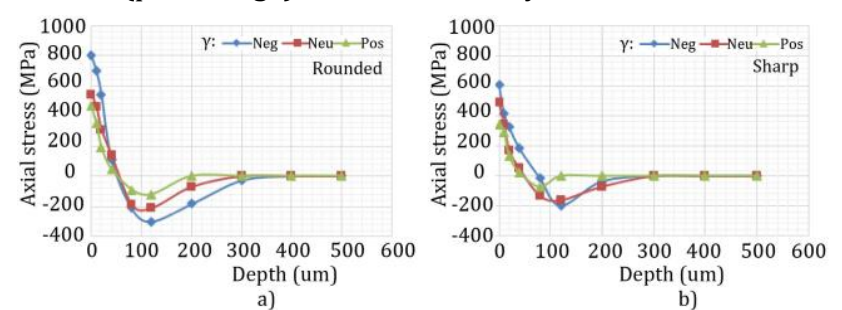

Fig. 7. Residual stress distribution (a) rounded, and (b) sharp tool

The values of material drag and residual stress were correlated with above calculated energy results, and same changing tendency was found for the surface integrity and $E_{\text {con }}$ when varying the tool geometries. Regarding the energy consumed in 
specific cutting areas, $E_{\mathrm{s}}$ is considered to be the main source that affects the surface deformation as it decreases more obviously (from $2.25 \mathrm{~J} / \mathrm{mm}^{3}$ to $1.12 \mathrm{~J} / \mathrm{mm}^{3}$ ) comparing with the decreasing of $E_{p}$ (from $0.25 \mathrm{~J} / \mathrm{mm}^{3}$ to $0.18 \mathrm{~J} / \mathrm{mm}^{3}$ ) when the grain deformation depth decreases from $21 \mu \mathrm{m}$ to $11 \mu \mathrm{m}$ for rounded tools (Fig.6-c1 to Fig6-c3). Referring to the $E_{f}$, it is believed that it has no contribution to the subsurface deformation as it is an energy mainly consumed between chips and tool rake face which is more related to the chip morphology.

When comparing the results from Fig. 6-c2 (rounded tool) and Fig. 6-c5 (sharp tool), which with the same $\gamma$ but different $R$, it could be found that the difference in $E_{p}$ leads to different grain deformation depth. This could also be validated by the comparison of Fig.6-c1 and Fig.6-c4, as well as Fig.6-c3 and Fig.6c6, which emphasized the contribution of $E_{p}$ on material deformation.

Thus, it could be concluded that the consumed energy $\left(E_{c o n}\right)$ is highly related to the adopted cutting parameters and could be employed as a feature to evaluate the machined surface integrity while both shear energy $\left(E_{\mathrm{s}}\right)$ and ploughing energy $\left(E_{p}\right)$ are playing play significant roles. More importantly, it illustrated that the proposed method could quickly reveal the inner relationship between the used cutting parameters and resulting surface damage level.

\subsection{Energy threshold for workpiece damage}

When considering the above conclusion that the level of surface damage could be related by the input energy $\left(E_{\mathrm{s}}, E_{\mathrm{p}}\right)$, this leads to the open question if the inverse relationship is valid: Given cutting parameters that yield the same input energy do they result in the same level of surface damage? If this is valid, it will open the possibility to define energy thresholds for ensuring that particular levels of surface damage are not exceeded; hence, no need to test various cutting parameters to attain this goal. To validate this, tests that result in three levels of input $E_{c o n}\left(E_{1}, E_{2}, E_{3}\right.$ - Fig.8), each of them with two different combinations of cutting parameters (Test A and Test B (Fig.8a)), have been carried out. For example, in Scenario 1, we compare Test A $(\gamma=$ neutral, $\left.v_{c}=150 \mathrm{~m} / \mathrm{min}\right)$ and test B $\left(\gamma=\right.$ negative, $\left.v_{c}=50 \mathrm{~m} / \mathrm{min}\right)$, similar energy partitions (Fig.8b) and surface deformation depth $(11 \mu \mathrm{m}$ for Test A and $10.5 \mu \mathrm{m}$ for Test B) are obtained. Comparable results have been found presented for different levels of energy for the other two scenarios (2 and 3) which result in same level of surface damage respectively.

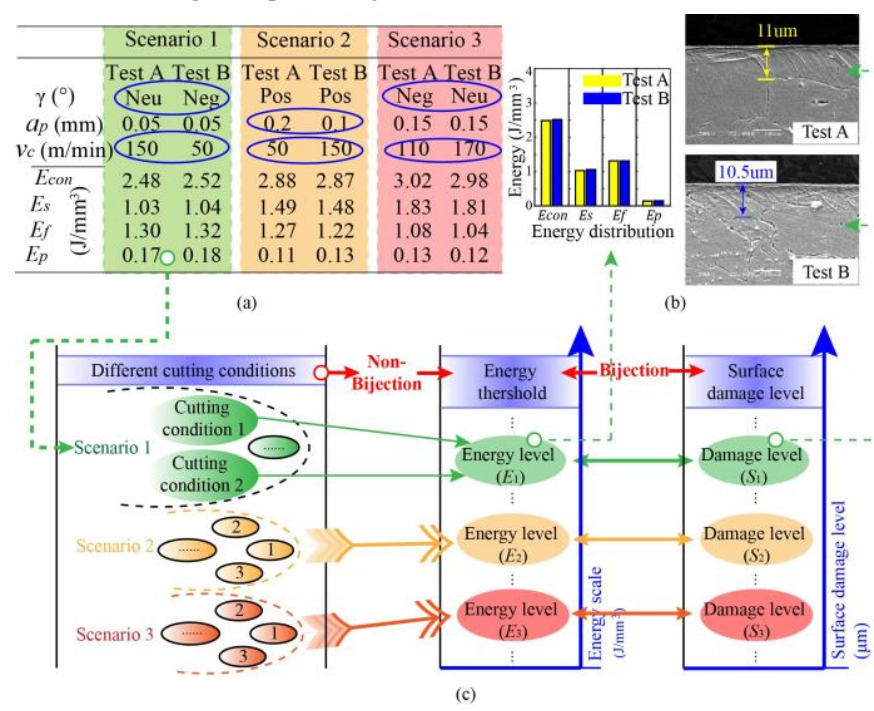

Fig. 8. Energy threshold for surface damage. Validation data sheet (a); Energy partition for scenario 1 (b); energy threshold evaluation (c)
In other words, as seen in Fig.8c, both cutting conditions (Test A and B) in Scenario 1 (green colour) yield the same energy level $E_{1}$ in cutting areas, which leads to an equivalent level of surface damage $S_{1}$. The relationship between cutting conditions and energy level is identified as non-bijection, which means that various combinations of cutting parameter could lead to the same input energy level. While bijective relationship is fixed between surface damage levels and input energy level $\left(E_{\mathrm{s}}, E_{\mathrm{p}}\right)$, showing that each certain level of surface damage (e.g. $S_{1}$ ) will correspond by one energy level (e.g. $E_{1}$ ) with the inverse relationship also being true. Examples for other energy levels (yellow colour for $E_{2}$ and red colour for $E_{3}-$ see Fig. 8c) following similar dependencies. It gives the perspective that a specific surface damage level could be controlled as long as the level of input energy keeps "constant" irrespective the cutting parameters combination used. Thus, an energy threshold for specific surface damage level could be identified with this proposed method.

This approach introduces a huge advantage for optimization and selection of cutting parameters resulting in specific surface integrity by studying the input energy levels during the cutting process rather than performing extensive machining trails with various cutting parameter combinations blindly.

\section{Conclusion and prospect}

This paper proposed a quick and effective PBCT method to carry out cutting tests that can evaluate energy threshold for machined surface integrity with specific damage level. The proposed method presents the following scientific breakthroughs and benefits.

- This research gives the ability to test materials rapidly without using conventional machine tools and it is applicable for materials in limited volume as well.

- The results indicate that machined surface integrity is highly correlated to the input total energy and partition in different cutting areas (shear and ploughing).

- An energy threshold could be defined for specific damage level of surface integrity, and used to evaluate material cutting performance irrespective of different cutting parameters.

- This proposed method also could be used to test other materials (such as composites, ceramics and plastic etc.) with less modification as it is not restricted to specific material.

\section{Acknowledge}

The authors acknowledge the support of Rolls-Royce., SECO Tools, and European Marie Curie Early-stage Research Program (The Integration of Novel Aerospace Technologies: INNOVATIVE).

\section{References}

[1] Liao ZR, et al (2018) On the influence of gamma prime upon machining of advanced nickel based superalloy. CIRP Ann Manuf Technol 67(1):109-112.

[2] Jawahir IS, et al (2011) Surface integrity in material removal processes: Recent advances. CIRP Ann Manuf Technol 60(2):603-626.

[3] Shang Z, et al (2019) On modelling of laser assisted machining: Forward and inverse problems for heat placement control. INT J MACH TOOL MANU 138: 36-50.

[4] AG A, et al (1985) Elastic and Plastic Fracture-Metals, Polymers, Ceramics, Composites, Biological Materials.

[5]Camposeco-Negrete C (2013) Optimization of cutting parameters for minimizing energy consumption in turning of AISI 6061 T6 using Taguchi methodology and ANOVA. J Clean Prod 53:195-203.

[6] Vingsbo $O$ (1985) Experimental studies of shear zones during chip formation in metal cutting. J. Phys. Colloq. 46:C5-371.

[7] Chandrasekaran $\mathrm{H}$, et al (2005) Modelling of material flow stress in chip formation process from orthogonal milling and split Hopkinson bar tests. Mach. Sci. Technol. 9(1):131-145.

[8] Shaw C (2005) Metal cutting principles, vol. 2. Oxford University press, New York [9] Budak E, et al (2016) Thermo-mechanical modeling of the third deformation zone in machining for prediction of cutting forces. CIRP Ann Manuf Technol 65(1):121-124

[10] Yen YC, et al (2004) A finite element analysis of orthogonal machining using different tool edge geometries. J. Mater. Process. Technol. 146(1): 72-81. 